\title{
EDITORIAL
}

\section{Organizing the Product Development Processes: Four Perspectives}

\author{
Eric Coatanéa ${ }^{*}$ \\ Department of Engineering Design and Production, School of Engineering, Aalto University, Helsinki, \\ Finland.
}

As stated by Zeng (Zeng, 2011), design comes from an environment, serves the environment, and changes the environment. Understanding the environment of a product is recognized by the research community to have a crucial impact on quality and success of products, services and complex systems.

In this context, the aim of the Journal of Integrated Design and Process Science (JIDPS) is to publish research findings covering transdisciplinary notions of design and process. The focus of the journal is aligned with three fundamental objectives which are; a) the understanding of design and process crossing boundaries of natural, human, and built environments, b) the principles, methods, and tools, and c) the applications of design and process science to engineering and social problems.

The present transaction is covering well those three lines of research by providing researches associated with:

1- The optimization of the scheduling and organization of the system development process,

2- The integration of product service system tools into the product development process,

3- The analysis of the consistency in the architecture of large scale systems,

4- The risk management in new product and service process,

The present issue is trying to understand and act on the environment in large of systems or services by understanding the processes associated with the design of those systems and services. The issue is also including research work about the principles, the methods and the tools.

The first paper, titled "A Combined Design Structure Matrix (DSM) and Discrete Differential Evolution (DDE) Approach for Scheduling and Organizing System Development Tasks Modelled using SysML" by Nonsiri et al, aims to develop a method supported by Artificial Intelligence approaches for automatically optimizing scheduling and organization of system development tasks. The purpose of this work is to support the scheduling and organization of tasks that can become very complex in system engineering process. Significant savings in term of development time can be made by providing a method that is optimizing the amount of feedbacks and iterations to the minimal level simply required for the successful development of the system. In this article, the authors are proposing to combine a computational approach (Discrete Differential Evolution) with Model Based Systems Engineering (MBSE) for minimizing iterations and reducing lead-time development. The present article is contributing to recent research works using Design Structure Matrixes (DSM) and computational methods for visualizing and analysing systems engineering processes. The practical use of the proposed framework is demonstrated on the case study of a mobile robot developed for the Eurobot competition. The article also

\footnotetext{
*Corresponding author. Email: eric.coatanea@aalto.fi Tel: (+358) 503605273.
} 
discusses the possibility to use the current framework to analyse the impact of requirement changes on the scheduling of development tasks.

The second paper, titled "Integrating Product-Service System Tools into New Product Development Processes" by Ribeiro et al, addresses integration of Product-Service system tools in the product development process. The paper collects methods and tools, and suggests its application, with the ultimate target to allow companies to be more competitive and provide sustainable products and related services. The paper develops a systematic literature review and interprets available data on PSS. The paper classifies the PSS practices according to the New Product Development phases in which they could be applied. The paper finally illustrates the application of PSS practices on a practical case study.

The third paper, titled "A Feasibility Study of the Ontology Driven Executable Systems Architecture Framework" by Khan et al, develop a feasibility study of a framework developed to manage the consistency in the architecture of large-scale system. It automatically generates a Coloured Petri nets (CPN) model from OWL ontology with SWRL rules embedded. The paper highlights the fact that the Guard conditions alone cannot always fulfil the requirements of operational rules mapping. It suggests mapping SWRL rules to arcs inscriptions in the CPN model. The overall transformation process helps in verifying a user's requirements, specified in SWRL rule, by simulating them in the corresponding automatically generated CPN model. The framework is exemplified using the case of a academic business processes in higher education institutions.

The fourth paper titled "Risk Management of Cyclically Recurring Project Activities of Product Realisation" by Berlec et al, presents an extended risk-analysis procedure for new product/service realisation projects. The usual risk analysis of project activities is based on evaluation of the probability that risk events occur and on evaluation of their consequences. Product/process realisation projects are recurring processes allowing also considering the recurring risk events. This is what is done in this paper. The project manager and team members also take into account the recurring risk events in risk management. A template was created in MS project environment. The project team used the template for testing the proposed methodology in a case study of realisation of a die-cast tool for manufacturing a car component.

The journal welcomes papers with systematic analysis of the design process and its associated methods and tools. Innovative and comprehensive applications are especially welcome.

\section{References}

Zeng, Y. (2011). Environment-Based Design (EBD). ASME Conference Proceedings, 2011(54860), 237250.

\section{Author Biography}

Dr. Eric Coatanéa received his double doctoral degree in Engineering design in 2005 from Helsinki University of Technology in Finland (nowadays Aalto University) and from University of West Brittany (France). He has also studied in Ecole Normale Supérieure and in INSA Toulouse. He was Marie-Curie Fellows from 2005 to 2007. He is Adjunct Professor in University of Grenoble and research fellow at Aalto University. He has been the leader and creator of the research group on Early development process and system engineering in Aalto and he is co-founder of a cooperative spin-off company exploiting the research results of his group in requirement engineering, modeling and simulation. His research interests include design and manufacturing, system thinking and system engineering, as well as eco-design, creativity and innovation. His current research focus is the development of support tools for the early design phases. 